\title{
Evidence of a local order in concentrated aqueous solutions of salts constituted by ions of different valences. $\mathrm{X}$-ray diffraction and Raman spectroscopy experiments
}

\author{
M. Alves Marques a,b,*, M.I. de Barros Marques ${ }^{\mathrm{a}, \mathrm{b}}$, M. Isabel Cabaço ${ }^{\text {a,b }}$, A.M. Gaspar ${ }^{\mathrm{a}, \mathrm{b}}$, \\ M.P.M. Marques ${ }^{\text {c,d }}$, A.M. Amado ${ }^{\text {c }}$, A.M. Amorim da Costa ${ }^{\mathrm{c}}$ \\ ${ }^{a}$ Departamento de Física, Instituto Superior Técnico, Universidade Técnica de Lisboa, Av. Rovisco Pais, 1049-001 Lisboa, Portugal \\ ${ }^{\mathrm{b}}$ Centro de Física Atómica da Universidade de Lisboa, Av.Prof. Gama Pinto, 2, 1649-003 Lisboa, Portugal \\ ${ }^{\mathrm{c}}$ Unidade ID Química Física Molecular, Departamento de Química, Universidade de Coimbra 3004-535 Coimbra, Portugal \\ d Departamento de Bioquímica, Universidade de Coimbra, 3001-401 Coimbra, Portugal
}

Available online 19 January 2007

\begin{abstract}
We revisit the fact that aqueous ionic solutions containing cations of higher valences than that of the anions display features in their X-ray patterns, which have been interpreted in terms of a medium range order between the cations.

Additional evidence is derived from Raman spectroscopy about the degeneracy removal in vibrational spectra of the nitrate anion. Inelastic neutron scattering experiments permitted to demonstrate the existence of characteristic modes in cation hydrates.

Model calculations, assuming independent vibrational species, are in agreement with a medium range order of the cations. We show also that a similar, but weaker, ordering arises if the anions are of higher valence than the cations.
\end{abstract}

(C) 2007 Elsevier B.V. All rights reserved.

\section{Introduction}

The solvation structure and the dynamics of ions in water play an important role in many processes that occur in a large variety of aqueous solutions. They are also relevant to many biological phenomena because biological structures are composed mainly of water. The existence of medium range correlations in the positions of heavy ions in concentrated aqueous solutions was suggested, long ago, by Prins [1] to interpret X-ray diffraction experiments in a number of different (liquid) electrolyte solutions. He discovered in photographic patterns of aqueous solutions with different concentrations of the same salt a moving ring which was interpreted by the existence of a superarrangement in these liquids. In a sequence of Raman investigations [2] two authors of this article studied

\footnotetext{
* Corresponding author. Centro de Física Atómica da Universidade de Lisboa, Av.Prof. Gama Pinto, 2, 1649-003 Lisboa, Portugal. Tel.: +351 217904879; fax: +351217954288.

E-mail address: marques@cii.fc.ul.pt (M.A. Marques).
}

the structure of a large ensemble of concentrated aqueous solutions by X-ray diffraction. These results [3] permitted to elaborate some conclusions, corroborated during ulterior investigations, which are summarised in the following points:

1. The existence of a maximum in the profile of the diffracted intensity is observed at $Q \sim 1 \AA^{-1}$ (first sharp diffraction peak or prepeak) in concentrated aqueous solutions of salts constituted by ions of different valences. For a pair of quasiisomorphous aqueous solutions, containing the same ions of low valence (e.g. $\mathrm{LaCl}_{3}$ and $\mathrm{AlCl}_{3}$ solutions), the intensity of the first sharp diffraction peak is larger for the solution with the ion of higher valence that exhibits the larger scattering power (Fig. 1). Even for a light ion (e.g. $\mathrm{BeCl}_{2}$ solutions) a prepeak can be detected.

2. The scattering of the ion of smaller valence, the counter ion, has a significant influence on the intensity of the prepeak: if the counter-ion is not included in the solvation shell of the ion of larger valence (e.g. $\mathrm{LaBr}_{3}$ and $\mathrm{LaCl}_{3}$ solutions), its scattering has a destructive interference in the intensity of the 


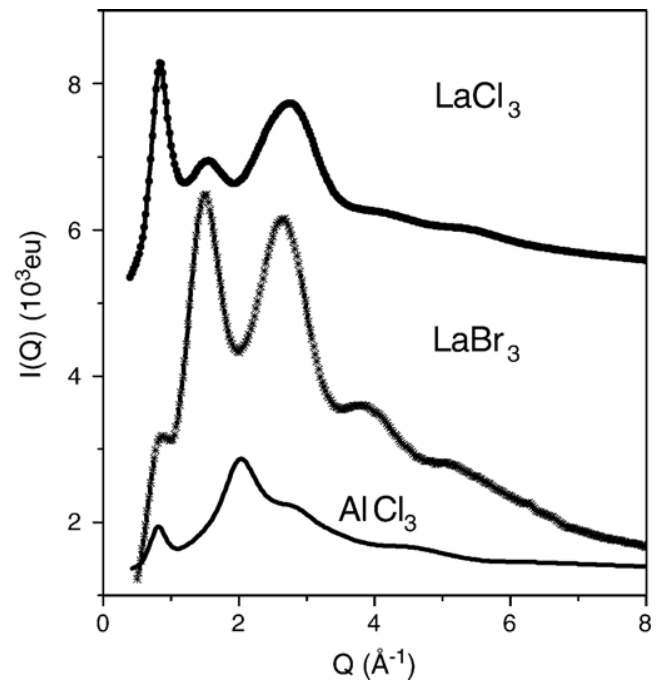

Fig. 1. X-ray diffraction patterns of $3.03 \mathrm{~mol} \mathrm{dm}^{-3} \mathrm{LaCl}_{3}$ (shifted $+4500 \mathrm{eu}$ ), $2.73 \mathrm{~mol} \mathrm{dm}^{-3} \mathrm{LaBr}_{3}$ and of $2.86 \mathrm{~mol} \mathrm{dm}^{-3} \mathrm{AlCl}_{3}$ (shifted +1000 eu) aqueous solutions.

prepeak (Fig. 1). However, if the counter-ion is included in the solvation shell (e.g. $\mathrm{InBr}_{3}$ and $\mathrm{InCl}_{3}$ solutions), its scattering power exhibits a constructive interference [4].

3. A proportionality between the position of the prepeak and the third root of the salt concentration is detected (Fig. 2). This fact was observed, qualitatively, by Prins [1].

4. A comparison between the profiles of the observed X-ray diffraction intensity with calculated profiles obtained from molecular models (see below) suggests, with some plausibility, the existence of a well-defined positional correlations of the ions of the larger valence in concentrated aqueous solutions [4-14].

5. The positions of the ions of larger valence are assumed to fluctuate around the sites of the first neighbours in a facecentred cubic lattice. ${ }^{1}$ The other neighbouring ions are distributed in a uniform continuum. Only stoichiometric distributions of ions were considered.

This distribution seems to be a consequence of the intense electrostatic repulsion between the ions of largest valence compared with that of the counter-ions and corresponds apparently to the configuration of minimal free energy (of Gibbs) of the liquid [5]. The existence of medium range ion-ion correlations at distances of the order of $8-9 \AA$ in very concentrated aqueous solutions has been discussed by many other authors [15-29].

In this article the authors intend to emphasize that the existence of this local order of cations can:

a) explain satisfactorily a partial lift of degeneracy of the vibrational spectrum of the nitrate anion in some solutions of

\footnotetext{
${ }^{1}$ As the authors consider as relevant only the positional correlations between the central ion and first neighbours, the cubic and the hexagonal closest packings do not lead to different models at this level.
}

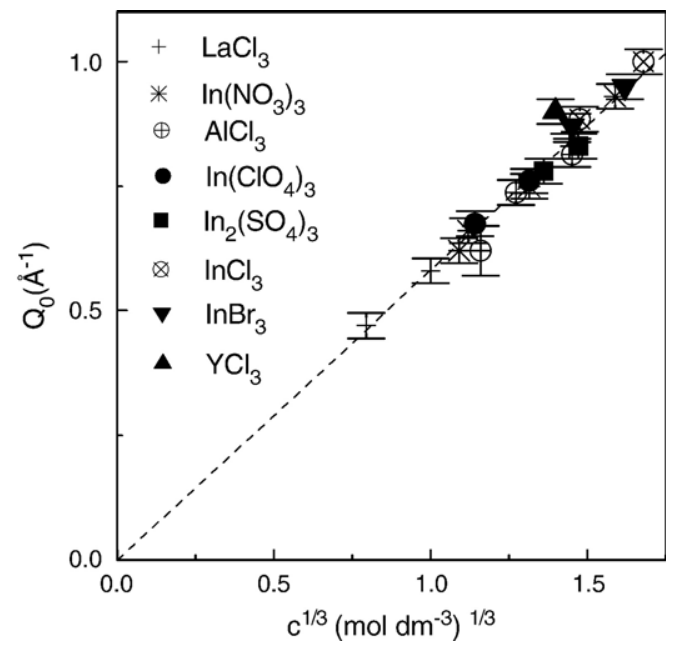

Fig. 2. Evolution of the prepeak position $Q_{0}$ with the molar concentration of aqueous solutions of inorganic salts of trivalent cations. The slope of the straight line was obtained assuming that the cations (in this case the ions of larger valence) are ordered in a local face-centred cubic packing.

metallic cations without assuming necessarily the existence of cation-anion pairs,

b) suggest that there is no coupling between vibrational spectra of cation hydrates in concentrated aqueous solutions of halides of aluminium,

c) suggest, once more [10], that medium range order can be determined by the anions if they are the ions of larger valence.

\section{Experiments and data reduction}

The aqueous solutions were prepared by weight, dissolving commercially available salts in bidistilled water. Solutions of $\mathrm{AlBr}_{3}$ and $\mathrm{AlCl}_{3}$ in deuterium oxide were prepared, too. Hydrated and anhydrous $\mathrm{AlCl}_{3}$, hydrated $\mathrm{AlBr}_{3}$, deuterium oxide $\left(\mathrm{D}_{2} \mathrm{O}>99.75 \%\right)$ and $\mathrm{NH}_{4} \mathrm{NO}_{3}$ were purchased from Merck, while anhydrous $\mathrm{AlBr}_{3}$ and hydrated $\left.\mathrm{Y}_{(} \mathrm{NO}_{3}\right)_{3}$ were obtained from Aldrich. The solutions of $\mathrm{InBr}_{3}, \mathrm{LaBr}_{3}, \mathrm{LaCl}_{3}$ and $\mathrm{Li}_{2} \mathrm{SO}_{4}$ and anhydrous $\mathrm{AlCl}_{3}$ were prepared from their

Table 1

Concentration and density of the aqueous solutions investigated

\begin{tabular}{lrll}
\hline Aqueous solution & $n$ & $\mathrm{~d}_{20}{ }^{\circ} \mathrm{C}\left(\mathrm{g} \mathrm{cm}^{-3}\right)$ & Concentration $\left(\mathrm{mol} \mathrm{dm}^{-3}\right)$ \\
\hline $\mathrm{AlBr}_{3}$ & 18 & 1.586 & 2.67 \\
$\mathrm{AlBr}_{3}$ & $18\left(\mathrm{D}_{2} \mathrm{O}\right)$ & 1.701 & 2.71 \\
$\mathrm{AlCl}_{3}$ & 18 & 1.309 & 2.86 \\
$\mathrm{AlCl}_{3}$ & $18\left(\mathrm{D}_{2} \mathrm{O}\right)$ & 1.394 & 2.82 \\
$\mathrm{InBr}_{3}$ & 14 & 1.896 & 3.06 \\
$\mathrm{InCl}_{3}$ & 14 & 1.556 & 3.22 \\
$\mathrm{LaBr}_{3}$ & 17 & 1.893 & 2.73 \\
$\mathrm{LaCl}_{3}$ & 16 & 1.634 & 3.03 \\
$\mathrm{Y}_{\left(\mathrm{NO}_{3}\right)_{3}}$ & 11 & 1.669 & 3.31 \\
& 15 & 1.509 & 2.89 \\
& 25 & 1.392 & 1.92 \\
$\mathrm{~K}_{3} \mathrm{PO}_{4}$ & 51 & 1.227 & 1.03 \\
$\mathrm{Li}_{2} \mathrm{SO}_{4}$ & 12 & 1.618 & 3.80 \\
& 19 & 1.220 & 2.70 \\
$\mathrm{NH}_{4} \mathrm{NO}_{3}$ & 30 & 1.151 & 1.78 \\
& 6.7 & 1.184 & 5.92
\end{tabular}


hydrated salts from Merck. The composition of the aqueous solutions investigated was monitored by chemical analysis (see Table 1).

The aqueous solutions investigated by Raman spectroscopy were filtered through nitrocellulose membranes, prepared in our laboratory, in order to reduce the background in the Raman spectra due to impurities of the solution. The presence of HDO or ordinary water in the deuterium oxide solutions was controlled via the intensity of the Raman spectra in the region of the corresponding intramolecular bands.

For the inelastic neutron scattering experiments the aqueous solutions in deuterium oxide were prepared and kept under argon environment in order to avoid any hydrogen contamination. Vitrification of the $\mathrm{AlCl}_{3}$ and $\mathrm{AlBr}_{3}$ solutions was achieved by immersing the filled sample container in liquid nitrogen.

The X-ray diffraction patterns of the aqueous solutions were obtained at the European Synchrotron Radiation Facility (ESRF, Grenoble) on the high energy beamline ID15b using monochromatized radiation of high energy $(88.54 \mathrm{keV}$, $0.140 \AA$ ). The samples were studied by transmission in layers of $2 \mathrm{~mm}$, contained in plane parallel cells between kapton windows $(25 \mu \mathrm{m})$ at room temperature. As for the X-ray diffraction patterns of the glassy samples, obtained at $25 \mathrm{~K}$, a closed cycle He refrigerator was used. The vacuum chamber had mylar windows $(100 \mu \mathrm{m})$ for the incident and scattered $\mathrm{X}$-rays.

The detection system was a MAR online image plate scanner $(2300 \times 2300$ pixels, pixel size $0.15 \mathrm{~mm})$. The onedimensional diffraction patterns were obtained by integration of the diffraction rings of the $2 \mathrm{D}$ patterns. In order to gather information over the relevant $Q$ domain the X-ray diffraction patterns were acquired at two different distances: $320 \mathrm{~mm}$ and $960 \mathrm{~mm}$ from the sample to the detector. A tube filled with helium was used to reduce significantly the contribution from air scattering to the measurements at the longest path between the sample and the detector.

Corrections were made for empty container, air (and helium) scattering contributions, absorption and geometry.

For all the atoms $\left(\mathrm{Li}, \mathrm{N}, \mathrm{O}, \mathrm{S}, \mathrm{Cl}, \mathrm{Br}, \mathrm{Y}, \mathrm{La}\right.$ in $\mathrm{Li}_{2} \mathrm{SO}_{4}, \mathrm{Y}$ $\left(\mathrm{NO}_{3}\right)_{3}, \mathrm{LaCl}_{3}$ and $\left.\mathrm{LaBr}_{3}\right)$ and ions $\left(\mathrm{Al}^{3+}, \mathrm{Cl}^{-}, \mathrm{Br}^{-}, \mathrm{In}^{3+}\right.$ in $\mathrm{AlCl}_{3}, \mathrm{AlBr}_{3}, \mathrm{InCl}_{3}$ and $\mathrm{InBr}_{3}$ ) the coherent scattering factors $f_{j}$ were taken from X-ray tables [30] and corrected for anomalous scattering [31]. Incoherent scattered intensities corrected for the Breit-Dirac recoil factor were calculated from Pálinkás and Radnai [32] except those from Pálinkás [33] for the Y atom and from Cromer and Mann [34] for the $\mathrm{Br}^{-}$ion. For $\mathrm{In}^{3+}$ an extrapolation was done from the In atom [35] based on Freeman and Watson [36]. For water the coherent scattering factors and the incoherent scattered intensity from Hajdu were used [37]. The corrected intensities were scaled to absolute units using the Krogh-Moe method [38].

Finbak's method, as reformulated by Warren [39], was applied to the experimental data and used to calculate the following total pair correlation function of the solution $g(r)$ :

$$
\begin{aligned}
g(r)= & 1 \\
& +\left(2 \pi^{2} r \rho_{\mathrm{e}} \sum_{u c} Z_{j}\right)^{-1} \int_{0}^{Q_{\max }} Q i^{\prime}(Q) e^{-b Q^{2}} \sin (r Q) \mathrm{d} Q
\end{aligned}
$$

where $\rho_{\mathrm{e}}$ is the average electron density of the solution, $Z$ the atomic numbers and $i^{\prime}(Q)$ the reduced intensity defined from the total intensity $I_{e u}(Q)$ as:

$i^{\prime}(Q)=\left(I_{e u}(Q)-\sum_{u c} f_{j}^{2}\right) / F^{2}(Q)=i(Q) / F^{2}(Q)$

where $F(Q)=\sum_{u c} f_{j} / \sum_{u c} Z_{j}$ is a sharpening factor. The units of composition $u c$, always refer to the ion of higher valence, which is the cation in all solutions, except in the cases of $\mathrm{K}_{3} \mathrm{PO}_{4}$ and $\mathrm{Li}_{2} \mathrm{SO}_{4}$.

In the sharpening factor $F(Q)$ the sum extends over all species in the solution (cation, anion and water molecules). Different values of $Q_{\max }$ were tested for both the KroghMoe method and the Fourier inversion. In the data analysis presented here we used $Q$ values up to $18 \AA^{-1}$. For that value of $Q_{\max ,}, b$ was set equal to $0.006 \AA^{2}$ in the modification function $e^{-b Q 2}$. The cutoff produces spurious peaks and decreases the spatial resolution, but this is not relevant for distance larger than $1 \AA$. A final correction has been made reducing the spurious oscillations in the pair correlation function $g(r)$ at small distances (less than $1 \AA$ ). The so-called pair correlation function $g(r)$ was then backtransformed in order to obtain the corrected dependent intensity $i(Q)$.

Raman spectra were obtained with a Jobin-Yvon spectrometer (T64000) using the $514.5 \mathrm{~nm}$ line of an $\mathrm{Ar}^{+}$laser in the classical $90^{\circ}$ scattering geometry and a CCD system was used as a detector. The overall spectral resolution was of $\sim 3 \mathrm{~cm}^{-1}$. Here the aqueous solutions were contained in borosilicate glass cylindrical cells of $10 \mathrm{~mm}$ inner diameter with the flat windows $2 \mathrm{~mm}$ thick.

Inelastic neutron scattering experiments were performed at the ISIS pulsed neutron source (Rutherford Appleton Laboratory, UK), using the TOSCA time-of-flight inverse-geometry spectrometer. Spectra were obtained by counting neutrons, from a white pulse, and scattered by the sample with a given final energy ( $\sim 4 \mathrm{meV}$ ) to detectors placed at a fixed scattering angle $\left(\sim 135^{\circ}\right)$. This single acquisition angle implies different values of momentum transfer associated to different values of energy transfer, with $Q^{2}$ values increasing almost linearly from $\sim 12.5 \AA^{-2}$ close to the elastic line to $120 \AA^{-2}$ for $200 \mathrm{meV}$ energy transfer.

The samples were placed in aluminium planar cells with $0.5 \mathrm{~mm}$ thick walls, protected against chemical attack from the aqueous solutions by nickel and gold coating. The sample thickness was set to $0.3 \mathrm{~mm}$ for solutions in ordinary water or to $3 \mathrm{~mm}$ for solutions in deuterium oxide. The cooling system was 


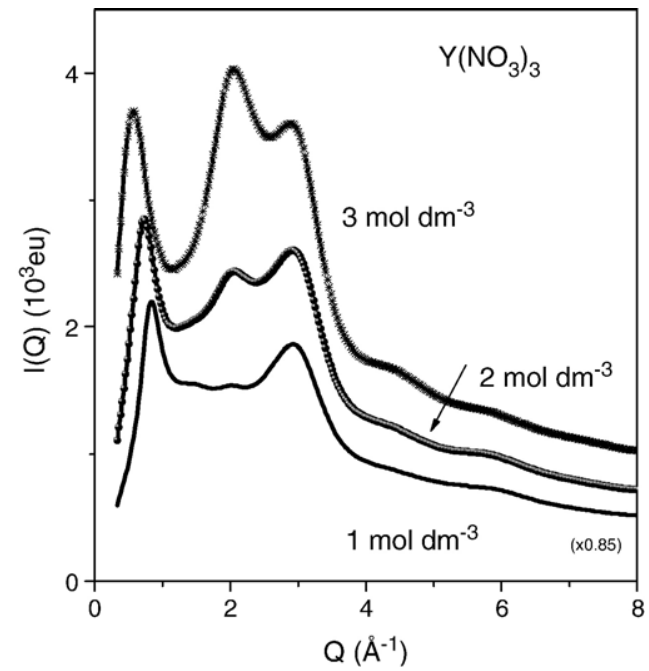

Fig. 3. X-ray diffraction patterns of $\mathrm{Y}\left(\mathrm{NO}_{3}\right)_{3}$ aqueous solutions of different concentrations.

a close-cycle He cryostat. All the spectra of the glassy state where obtained at $T \leq 25 \mathrm{~K}$.

Data processing included corrections for the energy distribution of the incoming neutron flux as well as conversion of the spectra for representation as function of energy transfer. This was done using standard programs available at ISIS. Scattering from the empty sample container was also taken into account.

\section{Results and interpretation}

\subsection{Lift of the degeneracy in the vibrational spectra of the nitrate anion}

The existence of medium range positional correlations in nitrate solutions as monitored by the presence of a first sharp maximum $\left(Q \sim 0.9 \AA^{-1}\right)$ in the X-ray diffraction patterns, is in agreement with several experimental results (Fig. 3). The lift of the vibrational degeneracy of the nitrate anion (Fig. 4) [40,41] in aqueous solutions of yttrium nitrate is shown in Fig. 5, which shows the evolution of the Raman spectra of the solution with increasing concentration from $1 \mathrm{~mol} \mathrm{dm}^{-3}$ up to $3.3 \mathrm{~mol} \mathrm{dm}^{-3}$. By comparing the calculated profiles obtained from tentative molecular models with the experimental one the authors concluded [14] that the existence of any nitrate anion in the first coordination shell of the yttrium cation does not seem to be compatible with the profile of the experimental intensity. It would be useful, certainly, to combine this result with a more

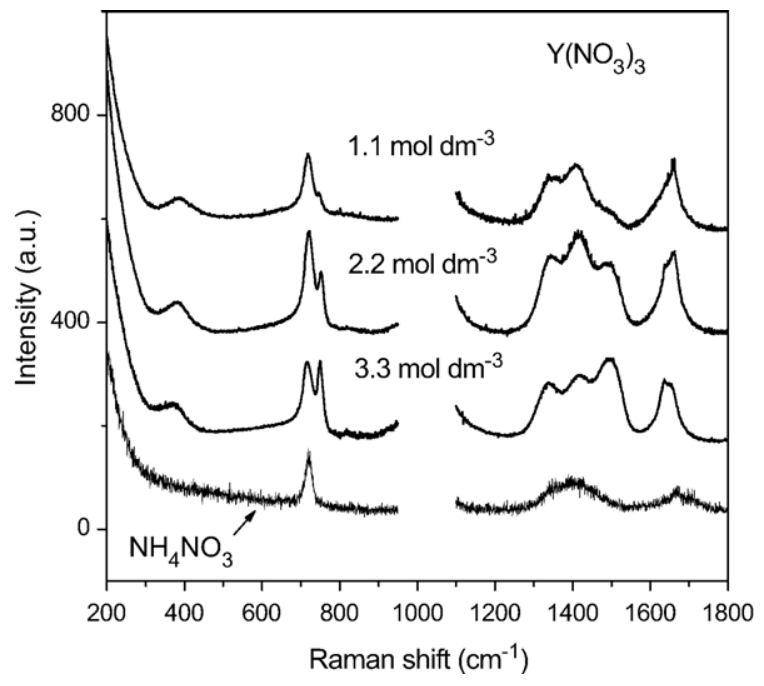

Fig. 5. Raman spectra of $\mathrm{Y}\left(\mathrm{NO}_{3}\right)_{3}$ aqueous solutions of different concentrations. The Raman spectra of a $5.92 \mathrm{~mol} \mathrm{dm}{ }^{-3} \mathrm{NH}_{4} \mathrm{NO}_{3}$ aqueous solutions is also displayed for comparison.

thorough study of the intramolecular dynamics of this anion, trying to predict quantitatively the removal of degeneracy in its vibrational spectra.

Taking into account the order in the distribution of the cations, electrostatic considerations suggested that the nitrogen nuclei of the nitrate anions are approximately collinear with two neighbour cations with an oxygen nucleus pointing to each one of them. This configuration was verified by a simulation, for details see Section 3.8, of the structure of concentrated aqueous solutions of yttrium nitrate (Fig. 6). In this situation the environment of each nitrate anion is not spherically symmetric and a destruction of the vibrational degeneracy is very plausible. When the solute concentration is lowered, the value of the electrostatic field is reduced. The environment of each nitrate becomes more symmetric and the degeneracy lift retains. The $\mathrm{X}$-ray intensity calculated from the model is larger than the observed one. This is interpreted here as due to the fact that in the structural model the cations were located in the geometric sites of a fcc lattice. Only small Gaussian fluctuations were considered without wide fluctuations, as it is described in Fig. 7 (see Section 3.4 Molecular models).

\subsection{Independence of the vibrational spectra of the neighbour aluminium hydrates}

The value of the frequency of the polarised band observed in the Raman spectrum of solutions of inorganic aluminium salts

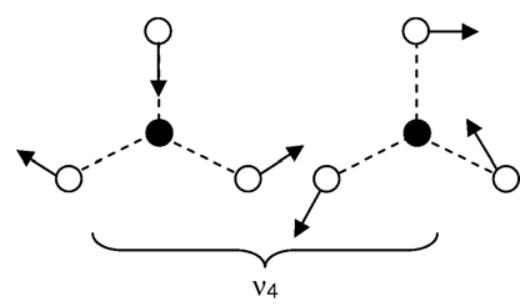

Fig. 4. Raman active degenerate vibrational modes of the nitrate anion [40]. 

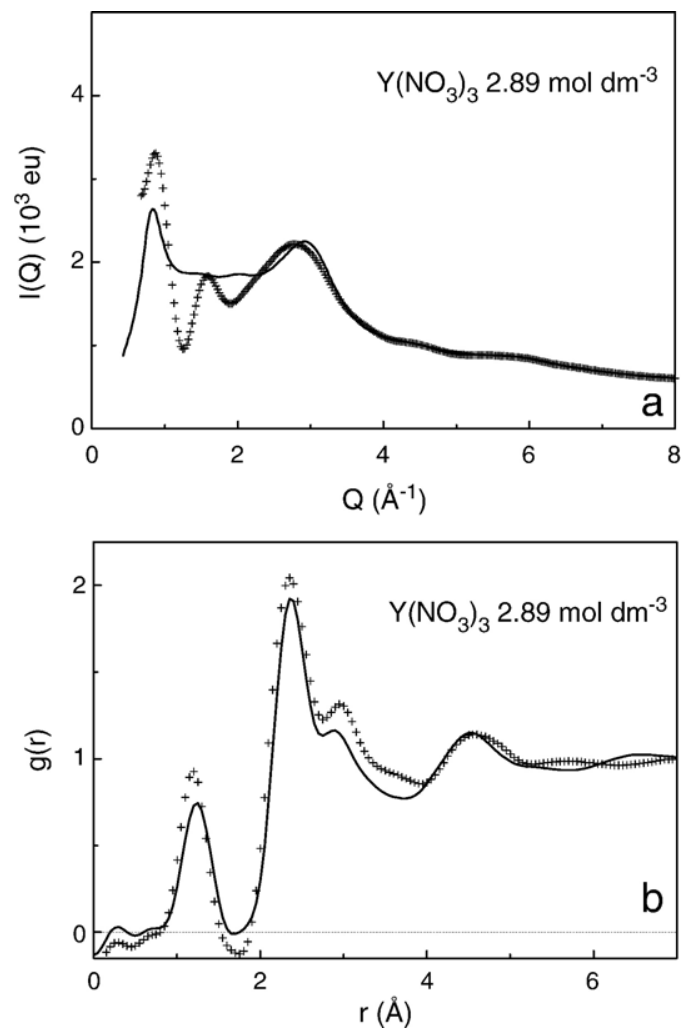

Fig. 6. Experimental (-) X-ray diffraction intensity (a) and pair correlation

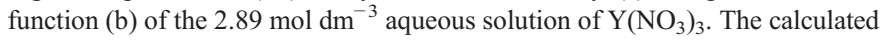
profiles ( $\cdots)$ were obtained from a structural model built in agreement with the hypothesis indicated in the text. The maximum and the minima displayed between 0.8 and $2 \AA^{-1}$ correspond to a local order of the yttrium cations located in the geometric sites of the fcc lattice (see Section 3.4 Molecular models).

in ordinary water is $\omega^{\mathrm{H} 2 \mathrm{O}}=524 \mathrm{~cm}^{-1}$ and its value when the solvent is deuterium oxide is $\omega^{\mathrm{D} 2 \mathrm{O}}=505 \mathrm{~cm}^{-1}$ which gives an isotopic shift of 1.038. If the motions of the coordinated water molecules in the vibrations corresponding to these bands were translational, the value of the isotopic shift should be the root mean square of the ratio of the total mass of the two water molecules $\left(m^{\mathrm{D} 2 \mathrm{O}} / \mathrm{m}^{\mathrm{H} 2 \mathrm{O}}\right)^{1 / 2}=1.054$ which does not agree with the experiment. So a coupling of the totally symmetric stretching vibration of the octahedral hydrate, where the water molecule performs only translational motions, must be considered.

Assuming the medium range order described above, an average cation-cation distance of $9 \AA$ is derived in this case for the saturated solution. Furthermore, the $\mathrm{X}$-ray experiments suggest the absence of cation-anion pairs. Thus, vibration couplings between the cation hydrates can be neglected and only couplings between the totally symmetric stretch of the hydrate and librational motions of the coordinated water molecules seem plausible.

The librational bands were obtained from inelastic neutron scattering (see Fig. 8). Here the observation of an additional activity, originated in many librational motions in the solutions, is notorious (Fig. 8). The authors assume that the librational motions of the water molecules, in the aluminium hydrates, are among these additional motions. The attribution of these bands to librations originates in the fact that the ratios of their frequencies in ordinary and in deuterated water are close to 1.4. These bands correspond, perhaps, to the three librational motions, rocking, wagging and twisting. The comparison between the inelastic neutron scattering intensities of two equimolecular solutions of aluminium chloride in ordinary and heavy water shows similar but scaled profiles. An important maximum located near $600 \mathrm{~cm}^{-1}$ in the ordinary water spectrum appears near $400 \mathrm{~cm}^{-1}$ in the spectrum of heavy water. A shoulder originating in a quasi coalescence of two bands in the region $\sim 750 \mathrm{~cm}^{-1}$ up to $\sim 880 \mathrm{~cm}^{-1}$ moves to a region that starts at $\sim 560 \mathrm{~cm}^{-1}$ and ends at $\sim 620 \mathrm{~cm}^{-1}$ for the solution in deuterium oxide. These correspondences suggest the definition of similar vibrational models for the two aluminium hydrates in light and in heavy water.

In our model four vibrational oscillations are considered. One, $v_{1}$, in essence the totally symmetrical pulsation of the octahedral aluminium hydrate, and three librational oscillations $v_{2}, v_{3}$ and $v_{4}$, of the coordinated water molecules of the same hydrate. They constitute the zero-approximation of the starting state. Between these four vibrational oscillations slight isotopic independent couplings are assumed. As the modifications of the values of the frequencies, resulting from the couplings are very small (the difference values are between $1 \%$ and $\sim 2 \%$ ) the authors suggest that the effects of partial modifications can be considered as additive. $\xi_{v_{1}}^{\mathrm{H}_{2} \mathrm{O}}, \xi_{v_{2}}^{\mathrm{H}_{2} \mathrm{O}}, \xi_{v_{3}}^{\mathrm{H}_{2} \mathrm{O}}$ and $\xi_{v_{4}}^{\mathrm{H}_{2} \mathrm{O}}$ are the values of the frequencies of the vibrations $v_{1}, v_{2}, v_{3}$ and $v_{4}$ in the zeroapproximation configuration before the effect of the couplings and $\xi_{v_{1}^{\prime}}^{\mathrm{H}_{2} \mathrm{O}}, \xi_{v_{2}^{\prime}}^{\mathrm{H}_{2} \mathrm{O}}, \xi_{v_{3}^{\prime}}^{\mathrm{H}_{2} \mathrm{O}}$ and $\xi_{v_{4}^{\prime}}^{\mathrm{H}_{2} \mathrm{O}}$ the values of the frequencies of

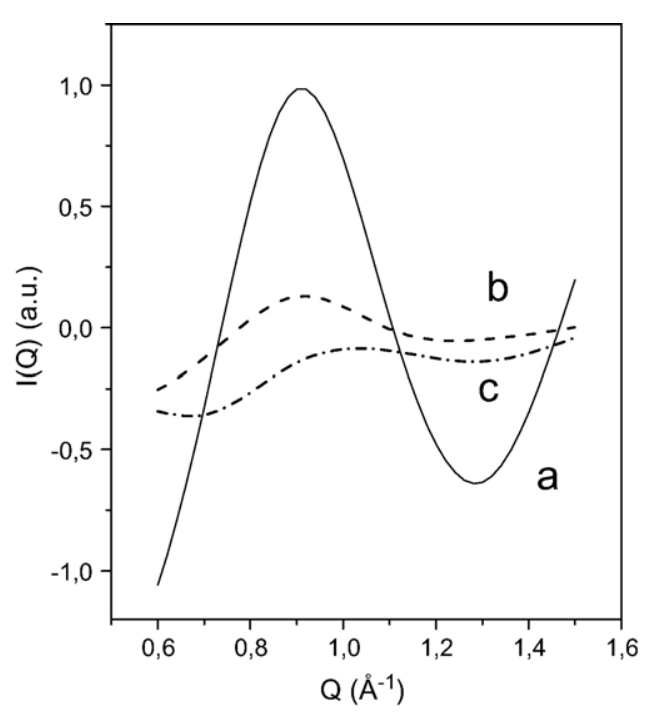

Fig. 7. Intensity profiles as obtained from different types of cubic close-packing structures: (a) Cubic close-packing where the scattering units are assumed in the geometric sites of a fcc lattice. The value of the distance between the first neighbours is $d=8.63 \AA$ and a Gaussian fluctuation of the value of the distance has a root mean square (rms) value $\delta=0.31 \AA$. (b) Cubic close-packing with the scattering units radially distributed on a spherical surface with the radius $\Delta=1.5 \AA$ A. Similar values of the distance $d$ between the first neighbours and of the rms value $(\delta)$ of the small fluctuations. (c) Cubic close-packing for a spherical surface of radius $\Delta=2.3 \AA$. 

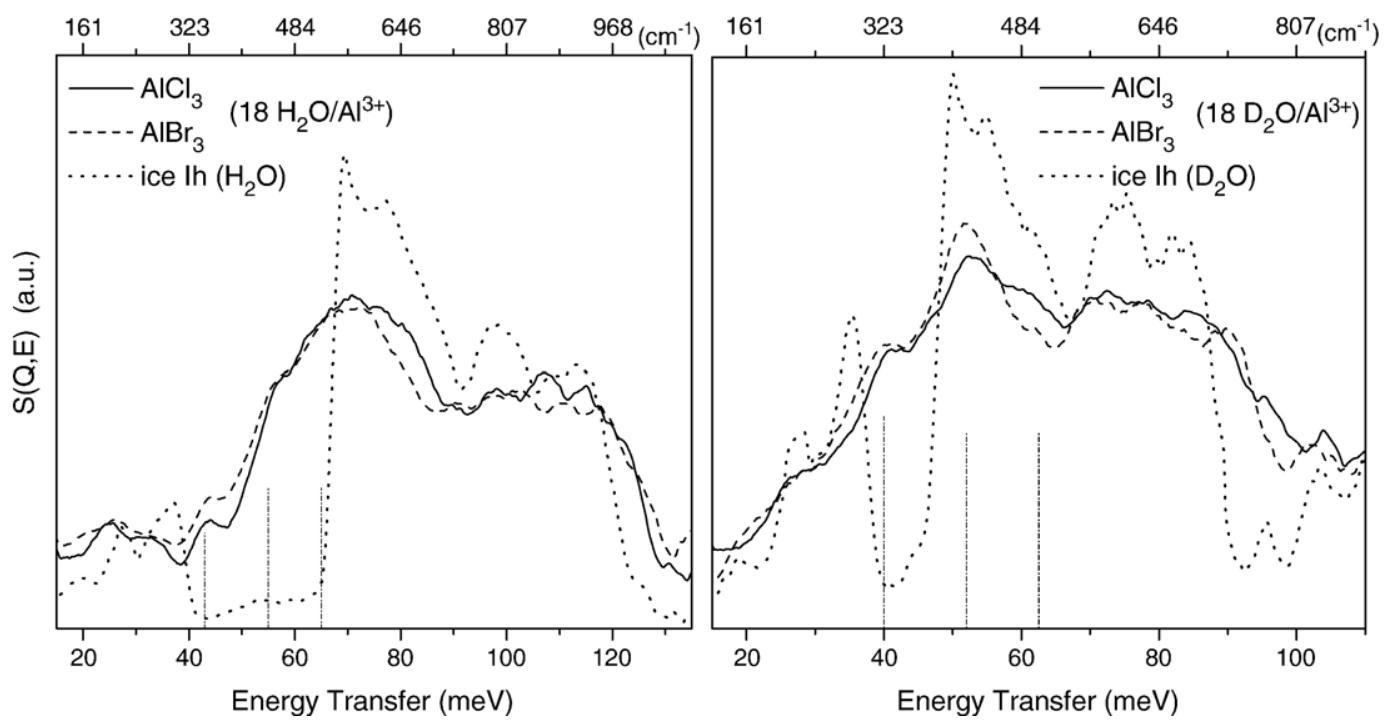

Fig. 8. Inelastic neutron scattering spectra of concentrated aqueous solutions of aluminium chloride and bromide in ordinary water and in deuterium oxide (18 water molecules per cation) in the glassy state $(T=25 \mathrm{~K})$. The spectra of pure ordinary water and pure deuterium oxide are displayed, too (dashed curves). The vertical lines indicate the frequency values 332, 440 and $524 \mathrm{~cm}^{-1}$ assigned to the active Raman bands of the aluminium hydrate [11].

the new vibrational modes, after couplings. For the coupling $v_{1}-v_{2}$, for instance, $\xi_{v_{1}^{\prime}}^{\mathrm{H}_{2} \mathrm{O}}, \xi_{v_{2}^{\prime}}^{\mathrm{H}_{2} \mathrm{O}}$ are obtained from the following equation:

$\xi_{v_{1}^{\prime}}^{2}, \xi_{v_{2}^{\prime}}^{2}=1 / 2\left(\xi_{v_{1}}^{2}+\xi_{v_{2}}^{2}\right) \pm \sqrt{1 / 4\left(\xi_{v_{1}}^{2}-\xi_{v_{2}}^{2}\right)+\chi\left(v_{1}, v_{2}\right) \xi_{v_{1}}^{2} \xi_{v_{2}}^{2}}$

where the coupling coefficients $\chi$ are considered to take similar values for hydrates with ordinary or with heavy water.

The values of the frequencies assigned to the four vibrational motions $v_{1}, v_{2}, v_{3}$ and $v_{4}$ before the couplings are given in Table 2. The values of the frequencies of the vibrational modes $v_{1}^{\prime}, v_{2}^{\prime}, v_{3}^{\prime}$ and $v_{4}^{\prime}$ after coupling, which are very similar to the zero-approximation motions, are given too (Table 2). These values were determined to obtain frequen-

Table 2

Assumed values of the frequency of the vibrational modes $v_{1}, v_{2}, v_{3}$ and $v_{4}$ before the intervention of the coupling coefficients $\chi_{i}$

\begin{tabular}{lll}
\hline & $\mathrm{H}_{2} \mathrm{O}$ & $\mathrm{D}_{2} \mathrm{O}$ \\
\hline$\xi_{v_{1}}$ & 532.6 & 505.5 \\
$\xi_{v_{1}^{\prime}}$ & 524.0 & 504.8 \\
$\xi_{v_{2}}$ & 602.9 & 426.4 \\
$\xi_{v_{2}^{\prime}}$ & 605.1 & 419.5 \\
$\xi_{v_{3}}$ & 803.9 & 568.6 \\
$\xi_{v_{3}^{\prime}}$ & 799.3 & 567.6 \\
$\xi_{v_{4}}$ & 874.2 & 618.2 \\
$\xi_{v_{4}^{\prime}}$ & 882.1 & 625.1 \\
\hline
\end{tabular}

And the frequencies of the vibrational modes $v_{1}^{\prime}, v_{2}^{\prime}, i_{3}^{\prime}$ and $v_{4}^{\prime}$ after the intervention of $\chi_{i}$. The value of the frequency of the oscillational mode that approaches the totally symmetrical pulsation, $v_{1}$, of the aggregate $\mathrm{Al}\left(\mathrm{H}_{2} \mathrm{O}\right)_{6}^{3+}$ was previously adjusted to $\xi_{v_{1}}^{\mathrm{H}_{2} \mathrm{O}}=524 \mathrm{~cm}^{-1}$ (the experimental value of the frequency of the polarized Raman band of the hydrate in ordinary water). The option $\chi\left(v_{1}, v_{2}\right)=0.006, \chi\left(v_{1}, v_{3}\right)=0.003$ and $\chi\left(v_{1}, v_{4}\right)=0.001$ lead to $\xi_{v_{1}^{2}}^{\mathrm{D}_{2} \mathrm{O}}=504.8 \mathrm{~cm}^{-1}$ (the experimental value is $505 \mathrm{~cm}^{-1}$ ). cies, after coupling, as close as possible to the experimental ones. The values of the coupling coefficients $\chi\left(v_{1}, v_{2}\right)=0.006$, $\chi\left(v_{1}, v_{3}\right)=0.003, \chi\left(v_{1}, v_{4}\right)=0.001$ lead to $\xi_{v^{\prime}}^{\mathrm{H}_{2} \mathrm{O}}=524 \mathrm{~cm}^{-1}$ (experimental value $524 \mathrm{~cm}^{-1}$ ) and to $\xi_{v_{1}^{\prime}}^{\mathrm{D}_{2} \mathrm{O}}=504.8 \mathrm{~cm}^{-1}$ (experimental value $\left.505 \mathrm{~cm}^{-1}\right)$. The values $\chi\left(v_{2}, v_{3}\right)=0.003$, $\chi\left(v_{2}, v_{4}\right)=0.003$ and $\chi\left(v_{3}, v_{4}\right)=0.003$ cannot be checked with the same precision as $\chi\left(v_{1}, v_{2}\right), \chi\left(v_{1}, v_{3}\right)$ and $\chi\left(v_{1}, v_{4}\right)$ because the inelastic neutron spectra of the aqueous solutions are very broad.

In order to obtain a value for the isotopic shift of this polarized Raman band, it seems necessary to invoke the existence of a coupling with a librational oscillation, the frequency of which is smaller than the one of the totally symmetric vibration (in deuterium oxide) and larger in the ordinary water.

\subsection{Investigations on solutions of salts constituted by anions di- and trivalent combined with monovalent cations}

To study the influence of the electric charges of the anions on the structural arrangement of the solution, when the anion is the ion of larger valence, two experiments were performed. In fact the maximum of the prepeak intensity was observed in the region of low $Q$ values in concentrated aqueous solutions of potassium phosphate and lithium sulphate. These observations are in keeping with the hypothesis of a medium range correlation of the anions, the ions of larger valence. The small value of the intensity of the prepeak is perhaps a consequence of the distribution of the negative electric charge of these anions over a large spatial domain. This is in contrast to cations discussed above. The uncertainty of the $Q$ value, corresponding to the localisation of the maximum, is of the order of $0.05 \AA^{-1}$. So for these solutions $\left(\mathrm{K}_{3} \mathrm{PO}_{4}\right.$ and $\left.\mathrm{Li}_{2} \mathrm{SO}_{4}\right)$ the presence of the vestigial maximum of intensity may be considered, plausibly, as indicative of the existence of medium 

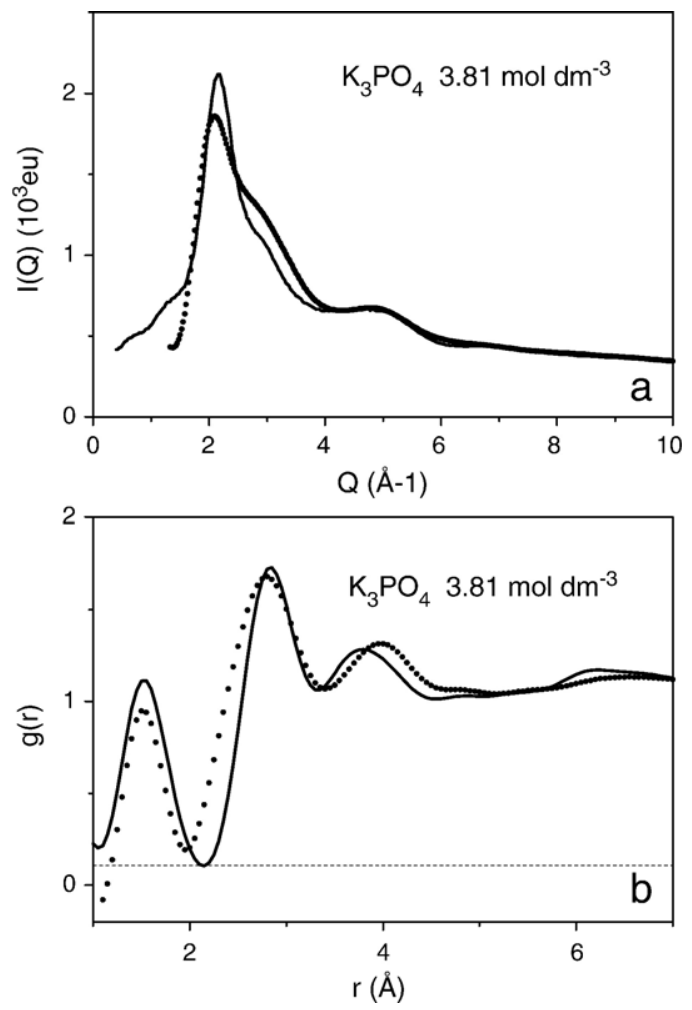

Fig. 9. Experimental (-) and calculated (*) X-ray diffraction intensity (a) and pair correlation function (b) of the $3.81 \mathrm{~mol} \mathrm{dm}^{-3}$ aqueous solution of $\mathrm{K}_{3} \mathrm{PO}_{4}$.

range positional correlations of the $\mathrm{PO}_{4}^{3-}$ and the $\mathrm{SO}_{4}^{2-}$ anions (Figs. 9 and 10).

\subsection{Molecular models}

We assume that the superposition of the X-rays scattered by the ions and molecules produces a total dependent intensity that may be computed by the Debye formula:

$i(Q)=\sum_{i \neq k} f_{i} f_{k} \frac{\sin \left(Q r_{i k}\right)}{Q r_{i k}}$

$f_{i}$ being the atomic or molecular scattering factors, $r_{i k}$ the distances between the atoms or molecules $i$ and $k$. The method has already been described [3-14], but small modifications are suggested here.

Two different internal configurations are considered in distinct regions: the first configuration, near an arbitrary central particle, is crystalline-type (discrete distribution); the other one, which starts at an adequate distance from the central particle and goes up to large distances, is simulated by a (random) uniform distribution. This uniform distribution may simulate, plausibly, the space averaged structure of the solutions as it is "seen" by the arbitrary central particle at large distances in the bulk of the liquid. The value $r_{\text {th }}$ of the threshold of this continuum is fixed as realistically as possible. Attention should be paid to the value of the scattered intensity produced by the uniform distribution. The intensity of the continuum, $i_{\mathrm{c}}(Q)$, ought to be smaller than the value obtained for the intensity from the discrete distribution, $i_{\mathrm{d}}(Q)$, in the range of $Q$ values relevant for the discussion of the models. The influence of a contribution originating from a region where the arrangement of the molecules and ions is uncertain ought to be minimized. In these models only a fraction of the first shell of the water molecules around the ions of high valence is considered in the discrete distribution [4]. The adopted fraction of the shell should include the molecules that face the central ion.

The intensities corresponding to some variants of the more plausible models were calculated in order to determine the influence of structural modifications compatible with the density of the concentrated solutions. None of the variants permitted to obtain an agreement as good as the one obtained with the model presented here. Taking into account the electrostatic interactions we assume that the counter-ions (ions of smaller electric charge) must be distributed at equal distances between the two ions of higher electric charge.

Another point must be emphasised: the location of the twelve ions of high valence that are first neighbours of a central ion deviate from the geometric sites of the face-centred cubic lattice. The thermal energy at room temperature causes positional fluctuations around these geometric sites. This is the reason why the expression liquid-type quasi-close packing was used [3-14]. When the intensity profile is calculated considering the twelve first neighbours fixed at the geometric sites an intense maximum (prepeak) followed by a deep well is obtained at $Q \sim 1 \AA^{-1}$ (Fig. 6a).

The final arrangement of scattering centres simulating a close-packing with fluctuations is constructed according to the following indication: instead of considering each pair of scattering sources at the close-packing distances, each source is simulated in six positions of equivalent weight. These positions result from shifts of the central particle, with respect to the second scatter. This hypothesis leads to a multiplicity of mutual distances. We tried two such shifts: the first, a

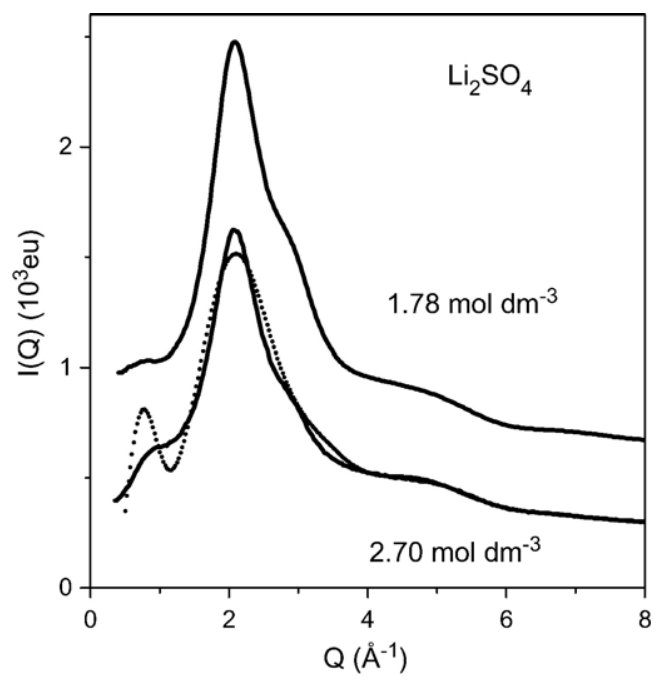

Fig. 10. Experimental (-) and calculated ( $\cdots)$ X-ray diffraction intensity (a) and pair correlation function (b) of the $2.70 \mathrm{~mol} \mathrm{dm}^{-3}$ and $1.78 \mathrm{~mol} \mathrm{dm}^{-3}$ aqueous solutions of $\mathrm{Li}_{2} \mathrm{SO}_{4}$. 
fluctuation of $1.5 \AA$, and then $2.3 \AA$. The obtained profiles are compared with the one that would be obtained if the first twelve neighbouring ions were considered in the geometric sites of the fcc lattice (Fig. 7).

The details of the procedure are as follows:

1. Only intensity profiles corresponding to discrete distributions $i_{\mathrm{d}}(Q)$ are compared.

2. The discrete intensity is calculated for $Q$ values near by $1 \AA^{-1}$ corresponding to the first maximum of $i_{\mathrm{d}}(Q)=\sin$ $(Q r) /(Q r)$ resulting from the interferences obtained from the scattering units (scattering factor 1) with only the first neighbours at distance of $9 \AA$ in a fcc lattice.

3. The scattering units are considered either in the geometric sites of the fcc lattice (Fig. 7a) or six regularly spaced positions were assumed regularly distributed on a spherical surface, with a radius $\Delta$, centred in these geometric sites (Fig. 7b and c). To obtain a convenient comparison between

Table 3

Structural data of the molecular model for $3.80 \mathrm{~mol} \mathrm{dm}^{-3} \mathrm{~K}_{3} \mathrm{PO}_{4}$ aqueous solution

\begin{tabular}{|c|c|c|c|c|}
\hline Correlations & $r$ & $\Delta r$ & $w$ & $r_{\text {th }}$ \\
\hline \multicolumn{5}{|l|}{ Anion-anion } \\
\hline $\mathrm{P}-\mathrm{P}$ & 8.53 & 1.16 & 3 & 11.23 \\
\hline \multirow[t]{2}{*}{$\mathrm{P}-\mathrm{O}$} & 1.54 & 0.03 & 8 & 9.05 \\
\hline & 7.32 & 0.74 & 24 & \\
\hline \multirow[t]{4}{*}{$\mathrm{O}-\mathrm{O}$} & 2.52 & 0.10 & 12 & 11.23 \\
\hline & 6.01 & 0.94 & 12 & \\
\hline & 7.59 & 1.10 & 48 & \\
\hline & 8.53 & 1.16 & 48 & \\
\hline \multicolumn{5}{|l|}{ Anion-cation } \\
\hline $\mathrm{P}-\mathrm{K}$ & 3.96 & 0.26 & 6 & 4.56 \\
\hline \multirow[t]{5}{*}{$\mathrm{O}-\mathrm{K}$} & 2.84 & 0.13 & 6 & 6.49 \\
\hline & 3.43 & 0.35 & 6 & \\
\hline & $\ldots$ & $\ldots$ & $\ldots$ & \\
\hline & 4.82 & 0.48 & 12 & \\
\hline & 5.29 & 0.52 & 6 & \\
\hline \multicolumn{5}{|l|}{ Anion-water } \\
\hline \multirow[t]{5}{*}{ P-water } & 3.03 & 0.29 & 12 & 6.00 \\
\hline & 3.96 & 0.29 & 8 & \\
\hline & $\ldots$ & $\ldots$ & $\ldots$ & \\
\hline & 6.03 & 0.52 & 16 & \\
\hline & 6.74 & 0.90 & 48 & \\
\hline \multirow[t]{5}{*}{$\mathrm{O}$-water } & 2.48 & 0.26 & 24 & 6.00 \\
\hline & 2.84 & 0.29 & 6 & \\
\hline & $\ldots$ & $\ldots$ & $\ldots$ & \\
\hline & 5.29 & 0.52 & 6 & \\
\hline & 5.63 & 0.55 & 48 & \\
\hline \multicolumn{5}{|l|}{ Cation-water } \\
\hline \multirow[t]{5}{*}{ K-water } & 2.81 & 0.29 & 12 & 7.00 \\
\hline & 3.03 & 0.29 & 12 & \\
\hline & $\ldots$ & $\ldots$ & $\ldots$ & \\
\hline & 6.56 & 0.65 & 24 & \\
\hline & 6.94 & 0.71 & 24 & \\
\hline \multirow[t]{5}{*}{ Water-water } & 2.81 & 0.29 & 12 & 8.00 \\
\hline & 3.00 & 0.29 & 12 & \\
\hline & $\ldots$ & $\ldots$ & $\ldots$ & \\
\hline & 6.94 & 0.71 & 24 & \\
\hline & 8.53 & 0.84 & 24 & \\
\hline
\end{tabular}

Values of the mutual distances $r$, r.m.s. fluctuations $\Delta r$, number of particles $w$, and threshold of the continuum $r_{\text {th }}$ for each kind of correlation. Distances are in $\AA$. the profiles (in Fig. 7) the thirty six different values (with the appropriate weights) of the mutual distances are assembled in three classes as the more representative. Supplementary small Gaussian fluctuations $(\delta)$ around these three representative values are assumed.

The calculated total intensity, $I_{e u}(Q)$, in electron units, that corresponds to the molecular models, is presented in all figures from $0.7 \AA^{-1}$ up to high $Q$ values. Indeed for $Q<0.7 \AA^{-1}$ the calculated intensities are produced by molecular distributions beyond an horizon of $10 \AA$ around the central particle.

In the model of the $3.80 \mathrm{~mol} \mathrm{dm}^{-3} \mathrm{~K}_{3} \mathrm{PO}_{4}$ aqueous solution we assume the $\mathrm{PO}_{4}^{3-}$ anions with the phosphorus atoms at the centre of a tetrahedron and the four oxygen atoms in the vertices at a distance of $1.54 \AA$ from the phosphorus atom. The local order of the ions of largest electronic charge, described in Section 1, was assumed for the tetrahedral phosphate anions (a local face centred cubic lattice considered as limited to the first neighbours of each phosphate anion). The three potassium atoms as well as three of the twelve water molecules (per anion) are in the middle points between two phosphorus atoms. Three more water molecules are distributed over the tetrahedral holes of the lattice and the remaining water molecules are in the octahedral holes. The profile of the calculated intensity was obtained from the numerical data inserted in Table 3.

The presence of the vestigial maximum (Fig. 9a) may be considered as indicative of positional correlations of the anions, as referred to in Section 3.3. However, our structural models, even though they take into account the constructive anionanion interferences, are not able to describe a so small maximum as the one seen at $Q=1 \AA^{-1}$ in Fig. 10. The overlap with the left wing of the large maximum of intensity $\left(Q \sim 2.2 \AA^{-1}\right)$ is too strong.

\section{Conclusions}

We have presented additional evidence for the existence of a medium range order between ions in concentrated aqueous solutions. This order is essentially created by the ions of higher valence, very clearly in the case of cations and more weakly, due to their charge distributions, by anions. This view is in keeping with thermodynamic considerations.

The existence of a prepeak in a number of molten salts constituted by ions of different valences has been interpreted, as corresponding to an ionic configuration similar to the one suggested here for concentrated aqueous solutions of similar salts. However, this comparison requires further discussion.

\section{Acknowledgements}

The authors are pleased to thank Dr. Thomas Buslaps and Dr. Veijo Honkimaki for their assistance on beamline ID15b during the X-ray diffraction experiments at ESRF. Professor John Finney and Dr. John Tomkinson are acknowledged for the given facilities in ISIS (Rutherford Appleton Laboratory) and Dr. Alexandre I. Kolesnikov for his help during the inelastic neutron scattering experiments at ISIS. The authors thank Ms. 
Maria Clara Carreiro da Costa, who prepared and analysed the solutions investigated.

We thank 'Fundação Calouste Gulbenkian' and 'Energia de Portugal (EDP)' for having financed the acquisition of some equipment (a powerful $\mathrm{Ar}^{+}$laser) for the experiments. A. M. Gaspar acknowledges the financial support given by 'Fundação para a Ciência e Tecnologia'.

\section{References}

[1] J.A. Prins, R. Fonteyne, Physica 2 (1935) 1016;

J.A. Prins, R. Fonteyne, J. Chem. Phys. 3 (1935) 72 and references therein.

[2] A. da Silveira, M. Alves Marques, N. Macias Marques, Comptes Rendus Acad. Sci. (Paris) 252 (1961) 3983;

A. da Silveira, M. Alves Marques, N. Macias Marques, Mol. Phys. 9 (1975) 271 .

[3] M. Alves Marques, M.I. de Barros Marques, Proc. K. Ned. Akad. Wet., B 77 (1974) 286.

[4] M.I. de Barros Marques, M.I. Cabaço, M.A. de Sousa Oliveira, M. Alves Marques, Chem. Phys. Lett. 91 (1982) 222.

[5] M. Alves Marques, M.I. Cabaço, Chem. Phys. Lett. 123 (1986) 73; M. Alves Marques, M.I. Cabaço, Chem. Phys. Lett. E 124 (1986) 487.

[6] M. Alves Marques, M.I. Cabaço, M.A. Sousa Oliveira, Chem. Phys. Lett. 126 (1986) 551.

[7] M.I. Cabaço, M. Alves Marques, M.I. de Barros Marques, G. BushnellWye, M.M. Costa, M.J. de Almeida, L.C. Andrade, J. Phys., Condens. Matter 7 (1995) 7409.

[8] M.I. Cabaço, A.M. Gaspar, C.M. de Morais, M. Alves Marques, J. Phys., Condens. Matter 12 (2000) 2623;

M.I. Cabaço, A.M. Gaspar, C.M. de Morais, M. Alves Marques, J. Phys., Condens. Matter E 13 (2001) 2751.

[9] M. Alves Marques, M.I. Cabaço, M.I. de Barros Marques, A.M. Gaspar, C.M. de Morais, J. Phys., Condens. Matter 13 (2001) 4367.

[10] M. Alves Marques, M.I. Cabaço, M.I. de Barros Marques, A.M. Gaspar, J. Phys., Condens. Matter 14 (2002) 7427.

[11] A.M. Gaspar, M. Alves Marques, M.I. Cabaço, M.I. de Barros Marques, A.I. Kolesnikov, J. Tomkinson, J.C. Li, J. Phys., Condens. Matter 16 (2004) 6343.

[12] A.M. Gaspar, M. Alves Marques, M.I. Cabaço, M.I. de Barros Marques, T. Buslaps, V. Honkimaki, J. Mol. Liq. 110 (2004) 15.
[13] M. Alves Marques, M.I. de Barros Marques, M.I. Cabaço, A.M. Gaspar, M. Luisa de Almeida, J. Mol. Liq. 110 (2004) 23.

[14] M. Isabel Cabaço, M.I. de Barros Marques, M. Alves Marques, A.M. Gaspar, M.L. de Almeida, J. Mol. Liq. 117 (2005) 69 and references therein.

[15] A.K. Dorosh, A.F. Skryshevskii, Z. Strukt. him. 8 (1967) 348.

[16] R.A. Howe, W.S. Howells, J.E. Enderby, J. Phys. C. Solid State Phys. 7 (1974) L111.

[17] J.E. Enderby, Proc. R. Soc. London A 345 (1975) 107.

[18] G.W. Neilson, R.A. Howe, J.E. Enderby, Chem. Phys. Lett. 33 (1975) 284

[19] R. Caminiti, G. Licheri, G. Piccaluga, G. Pinna, Rev. Inorg. Chem. 1 (1979) 333.

[20] G. Licheri, G. Piccaluga, G. Pinna, J. Am. Chem. Soc. 101 (1979) 5438.

[21] J.E. Enderby, G.W. Neilson, Adv. Phys. 29 (1980) 323.

[22] A. Habenschuss, F.H. Spedding, J. Chem. Phys. 73 (1980) 442.

[23] A.K. Soper, J.E. Enderby, G.W. Neilson, Rep. Prog. Phys. 44 (1981) 595.

[24] G. Pálinkás, E. Kalman, Z. Naturforsch. 36a (1981) 1367.

[25] M. Magini, G. Paschina, G. Piccaluga, J. Chem. Phys. 76 (1982) 1116 and references therein.

[26] G.W. Neilson, J.E. Enderby, Proc. R. Soc. London A 390 (1983) 353.

[27] T. Iijima, K. Nishikawa, Chem. Phys. Lett. 115 (1985) 522.

[28] W. Meier, P. Bopp, M.M. Probst, E. Spohr, J.-L. Lin, J. Phys. Chem. 94 (1990) 4672.

[29] R. Mills, N.H. March, P.V. Giaquinta, M. Parrinello, M.P. Tosi, Chem Phys. 26 (1977) 237.

[30] A.J.C. Wilson (Ed.), International Tables for Crystallography C, 1992.

[31] C.T. Chantler, J. Phys. Chem. Ref. Data 29 (2000) 597; C.T. Chantler, J. Phys. Chem. Ref. Data 24 (1995) 71.

[32] G. Pálinkás, T. Radnai, Acta Crystallogr., A 32 (1976) 666.

[33] G. Pálinkás, Acta Crystallogr., A 29 (1973) 10.

[34] D.T. Cromer, J.B. Mann, Acta Crystallogr., A 24 (1968) 321.

[35] V.H. Smith Jr., A.J. Thakkar, D.C. Chapman, Acta Crystallogr., A 31 (1975) 391.

[36] A.J. Freeman, R.E. Watson, Acta Crystallogr. 15 (1962) 682.

[37] F. Hajdu, Acta Crystallogr., A 28 (1972) 250.

[38] J. Krogh-Moe, Acta Crystallogr. 9 (1956) 951; N. Norman, Acta Crystallogr. 10 (1957) 370.

[39] B.E. Warren, X-ray Diffraction, Addison-Wesley, 1968.

[40] J.P. Mathieu, Faraday Soc. Discuss. 9 (1950) 196.

[41] R.E. Hester, thesis Cornell University (1962). 